Andrei B. Borisov · Eduard I. Dedkov · Bruce M. Carlson

\title{
Differentiation of activated satellite cells in denervated muscle following single fusions in situ and in cell culture
}

Accepted: 30 March 2005 / Published online: 6 July 2005

(C) Springer-Verlag 2005

\begin{abstract}
Satellite cells represent a cellular source of regeneration in adult skeletal muscle. It remains unclear why a large pool of stem myoblasts in denervated muscle does not compensate for the loss of muscle mass during post-denervation atrophy. In this study, we present evidence that satellite cells in long-term denervated rat muscle are able to activate synthesis of contractile proteins after single fusions in situ. This process of early differentiation leads to formation of abnormally diminutive myotubes. The localization of such dwarf myotubes beneath the intact basal lamina on the surface of differentiated muscle fibers shows that they form by fusion of neighboring satellites or by the progeny of a single satellite cell following one or two mitotic divisions. We demonstrated single fusions of myoblasts using electron microscopy, immunocytochemical labeling and high resolution confocal digital imaging. Sequestration of nascent myotubes by the rapidly forming basal laminae creates a barrier that limits further fusions. The recruitment of satellite cells in the formation of new muscle fibers results in a progressive decrease in their local densities, spatial separation and ultimate exhaustion of the myogenic cell pool. To determine whether the accumulation of aberrant dwarf myotubes is explained by the intrinsic decline of myogenic properties of satellite cells, or depends on their spatial separation and the environment in the tissue, we studied the fusion of myoblasts isolated from normal
\end{abstract}

\footnotetext{
A. B. Borisov $(\square)$

Division of Pediatric Cardiology, Department of Pediatrics and Communicable Diseases, University of Michigan Medical School, Room 8200, MSRB III, Ann Arbor, MI 48109-0616, USA

E-mail: aborisov@med.umich.edu

Tel.: + 1-734-6479429

Fax: + 1-734-6151386

E. I. Dedkov · B. M. Carlson

Department of Cell and Developmental Biology, University of Michigan Medical School, Ann Arbor, MI 48109-0616, USA

B. M. Carlson

Institute of Gerontology, University of Michigan Medical School, Ann Arbor, MI 48109-0616, USA
}

and denervated muscle in cell culture. The experiments with a culture system demonstrated that the capacity of myoblasts to synthesize contractile proteins without serial fusions depended on cell density and the availability of partners for fusion. Satellite cells isolated from denervated muscle and plated at fusion-permissive densities progressed through the myogenic program and actively formed myotubes, which shows that their myogenic potential is not considerably impaired. The results of this study suggest that under conditions of denervation, progressive spatial separation and confinement of many satellite cells within the endomysial tubes of atrophic muscle fibers and progressive interstitial fibrosis are the important factors that prevent their normal differentiation. Our findings also provide an explanation of why denervated muscle partially and temporarily is able to restore its functional capacity following injury and regeneration: the release of satellite cells from their sublaminal location provides the necessary space for a more active regenerative process.

Keywords Skeletal muscle - Satellite cells . Myogenesis - Denervation-atrophy-regeneration

\section{Introduction}

During development and regeneration of skeletal muscle, thousands of mononucleated myoblasts fuse and differentiate into muscle fibers (reviews - Buckingham 2001; Sanger et al. 2004; Charge and Rudnicki 2004). A subpopulation of myoblasts resides beneath the basal lamina of the nascent muscle fibers and forms a pool of inactive myogenic stem cells, or satellite cells. In adult mammalian muscle, depending on species and anatomical characteristics, the number of satellite cells typically comprises $2-5 \%$ of the total number of myonuclei, and these cells can effectively activate the regenerative process following even minimal muscle injury (Carlson 1991; Schultz and McCormick 1993; Hawke and Garry 
2001; Morgan and Partridge 2003; Bischoff and Franzini-Armstrong 2004).

At the present time, only fragmentary information is available concerning the early responses of satellite cells to muscle atrophy of different etiologies. One of the most common and clinically relevant types of muscle pathology is post-denervation muscle atrophy. Denervation of adult muscle results in a rapid decline of its functional capacity and progressive atrophy of muscle fibers. It remains unclear why muscle tissue atrophies despite the presence of a large reserve of myogenic cells. Paradoxically, there is evidence that in denervated muscle the number of satellite cells may double or triple during the first few weeks following denervation (for discussion, see Vigue et al. 1997). Thus, understanding the behavior and properties of myoblasts in atrophying muscle is of great biological and clinical importance. Despite the fact that knowledge regarding the molecular pathways underlying myogenic commitment and differentiation of precursor cells during embryonic development has substantially increased during the last decade, much less is known as yet about the overall mechanisms that control the sequential events of regeneration during muscle atrophy. For example, the control of early stages of the regenerative response in denervated muscle is still poorly understood, and it still remains to be determined what stage of regeneration is most impaired during postdenervation muscle atrophy. Successful fusion of activated myogenic cells to one another is one of the critical events of myogenesis. The control of fusion between myoblasts, as well as myoblasts and myotubes in normal muscle development, remains perhaps the least well understood process in myogenic differentiation (Bischoff 1990, Chen and Olson 2004; Horsley and Pavlath 2004). At the same time, little is known about the fate of myoblasts and their capacity for normal fusion in denervated muscle.

Recent introduction in research practice of highly improved immunological reagents and advanced techniques of confocal microscopy have made it feasible to analyze the biology of muscle growth and regeneration at the single cell level. These techniques allow one to visualize satellite cells and perform a high resolution analysis of early stages of their involvement in cytodifferentiation of individual myofibers. The purpose of the present study was to investigate the involvement of satellite cells in formation of new myotubes in long-term denervated rat skeletal muscle with special reference to the process of their fusion.

\section{Materials and methods}

\section{Muscle denervation}

The experiments were conducted on adult (4 month old) male WI/HicksCar rats maintained in the animal facility at the Department of Biology, University of Michigan. In all experiments the animals were anesthetized with ether. The right leg of each rat was denervated by sectioning the sciatic nerve high in the thigh area. The sciatic nerve was tightly ligated with surgical silk in two places, cut between the sutures, and a $0.4 \mathrm{~cm}$ section of the nerve was removed. Both proximal and distal nerve stumps were implanted in the surrounding tissue as far away from each other as possible. This procedure allows a permanent and complete denervation of the lower leg (Carlson and Faulkner 1988). The animals were euthanized under ether anesthesia at 1, 2, 4 and 7 months after denervation. Tissue samples of the extensor digitorum longus and tibialis anterior muscles were taken from four animals for each time point and processed for immunohistochemical and electron microscopic analysis. The samples of fetal muscle tissue were taken from 19 day-old rat embryos.

\section{Immunohistohemistry and fluorescent microscopy}

Each muscle was divided into two parts, cryoprotected in a graded sucrose series, and embedded in molds for transverse and longitudinal sectioning in cryoprotective freezing medium TBS (Triangle Biomedical Sciences, Durham, North Carolina, USA). The samples were sectioned into $8 \mu \mathrm{m}$ sections through the equatorial area of each muscle and mounted in groups of four on histological slides. After the embedding medium was washed out, the tissue sections were immunolabeled using the technique of indirect immunofluorescence. Three to five slides at each time point were studied from each animal. Monoclonal antibodies to embryonic and sarcomeric isoforms of myosin, myogenin, NCAM and laminin were obtained from the Developmental Studies Hybridoma Bank (University of Iowa, Iowa City, IA, USA), Chemicon International Inc. (Temecula, CA, USA) and Novocastra Laboratories Ltd. (Newcastle upon Tyne, UK). Fluorescein-conjugated rabbit anti-mouse $\operatorname{IgG}$ (Sigma, St. Louis, MO, USA) was used as a secondary antibody for the detection of binding of monoclonal antibodies. TRITC-conjugated goat anti-rabbit IgG (Sigma, St. Louis, MO, USA) was used as a secondary antibody for visualization of binding of polyclonal primary rabbit antibodies. The antibodies were diluted according to the recommendations of the producers. The immunofluorescent reactions were examined with a $\mathrm{Ni}$ kon Optiphot fluorescence microscope equipped with an excitation filter at 470-490 nm and emission barrier filter at $510-560 \mathrm{~nm}$ wavelengths for fluorescein, and with an excitation filter at 510-560 $\mathrm{nm}$ and emission barrier filter at $590-615 \mathrm{~nm}$ for TRITC. Confocal analysis was performed with a Carl Zeiss LSM 510 Meta confocal microscope using the $\times 40$ and $\times 63$ objectives.

\section{Electron microscopy}

Samples of muscle tissue were prefixed immediately after excision from the limb in an ice-cold solution of $2.5 \%$ 
glutaraldehyde and $4 \%$ formaldehyde in $0.1 \mathrm{M}$ isoosmotic phosphate buffer, $\mathrm{pH} 7.4$ for $1 \mathrm{~h}$. The tissue was then cut into several smaller pieces and fixed for an additional $4 \mathrm{~h}$ at $3^{\circ} \mathrm{C}$ in fresh aliquots of fixative. After removal of fixative, the samples were washed three times for $15 \mathrm{~min}$ each in $0.1 \mathrm{M}$ phosphate buffer. The tissue was postfixed with $1 \% \mathrm{OsO}_{4}$ in $0.1 \mathrm{M}$ phosphate buffer for $1.5 \mathrm{~h}$ at $3^{\circ} \mathrm{C}$, washed again in the buffer solution three times (15 min each wash) and dehydrated through a graded ethanol series to absolute ethanol. After infiltration with epoxy resin, the samples were embedded in Spurr medium (Ted Pella, Inc., Redding, CA, USA). After polymerization of the medium in blocks, ultrathin sections were prepared using a Reichert-Young Ultracut E ultramicrotome and mounted on copper/rhodium mesh grids M150-CR (Electron Microscopy Sciences, Fort Washington, PA, USA). After staining with uranium acetate and lead citrate (Reynolds 1963), the sections were examined with a Philips CM100 electron microscope at an accelerating voltage of $60 \mathrm{kV}$.

\section{Cell culture}

Cell cultures of satellite cells have been prepared by digestion of the minced extensor digitorum longus and tibialis anterior muscles with $0.2 \%$ collagenase. The suspension of isolated cells was plated into $60 \mathrm{~mm}$ Petri dishes (Corning, Inc., USA) coated with Matrigel (Becton-Dickinson, Bedford, MA, USA) and containing $90 \%$ Eagle medium and $10 \%$ fetal calf serum (GIBCO, USA) at the plating densities $3.7 \times 10^{4}$ and $3.7 \times 10^{3}$ cells per a dish. To induce cell differentiation, fetal calf serum was replaced with equine serum on day 5 of cultivation. The technique of cell culture has been described in detail in our earlier publication (Borisov et al. 1985). For detection of the expression of myogenic markers, the cells were grown on coverslips, fixed with $2 \%$ paraformaldehyde and immunofluorescently labeled for microscopic analysis as described above.

\section{Results}

Taking into account the similarity of cell differentiation pathways during normal development and regeneration, we used immunolabeling for the following protein markers of myogenesis: (1) MyoD and myogenin, the myogenic regulatory factors expressed by committed myoblasts before and following fusion (Yablonka-Reuveni and Paterson 2001; Paoni et al. 2002; Pownall et al. 2002; Emerson and Hauschka 2004), (2) the embryonic isoform of myosin expressed in nascent muscle fibers (Paoni et al. 2002; Matecki et al. 2004), and (3) the isoform of neural cell adhesion molecule (NCAM) typical of activated myogenic cells and early myotubes (Mesires and Doumit 2002; Crameri et al. 2004).

Studies of longitudinal sections of denervated muscle using double immunofluorescent labeling and Nomarski interference optics demonstrated the presence of binucleated and trinucleated myotubes intensely immunopositive for the myogenic regulatory factors (Fig. 1a-c). The cellular localization of MyoD protein was associated with myonuclei (Fig. 1), and myogenin had a very similar pattern of predominantly nuclear expression (not shown, the localization was identical to the pattern
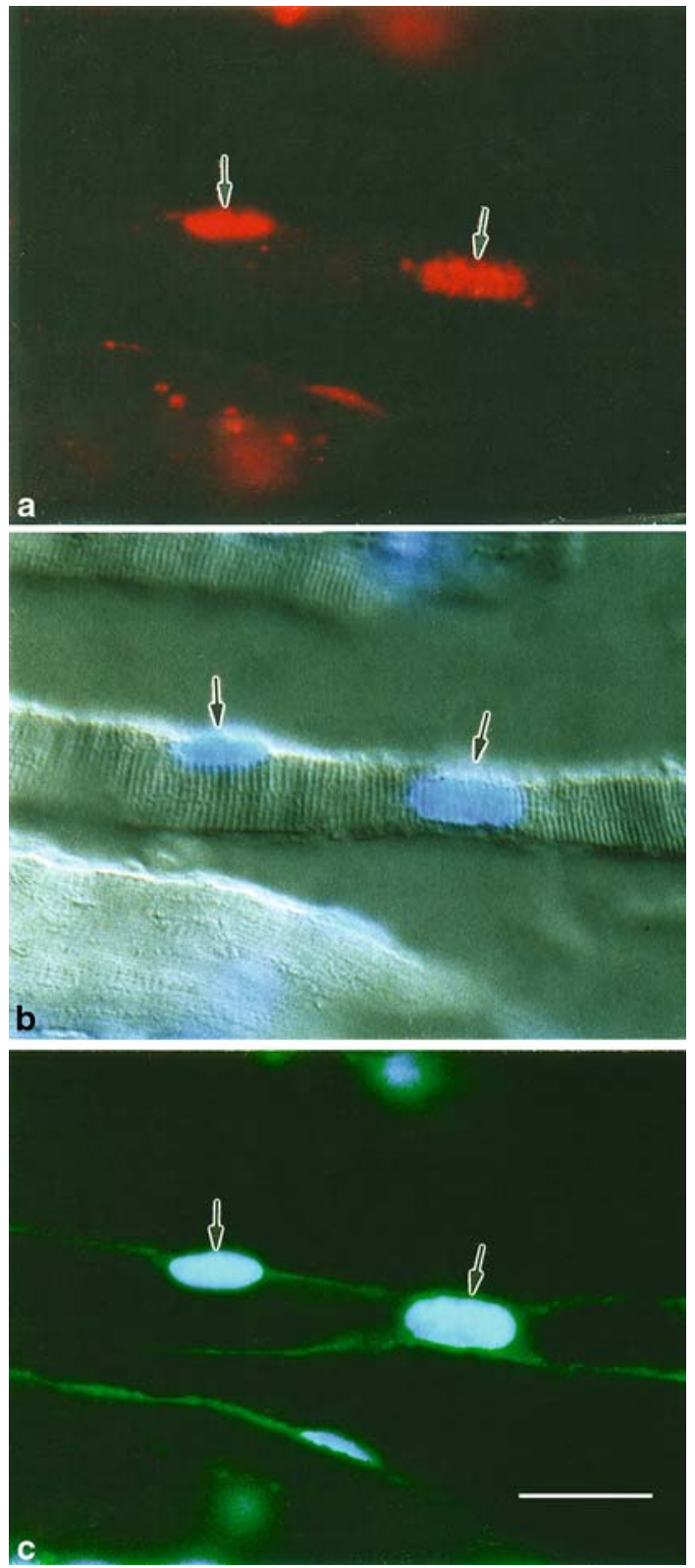

Fig. 1 a-c Expression of myogenic regulatory factors in small myotubes in long-term denervated skeletal muscle. a Immunolabeling for MyoD (red fluorescence). b Nuclear DNA staining with bisbenzimide (blue fluorescence) combined with Nomarski interference contrast. c Nuclear DNA staining with bisbenzimide combined with immunolabeling of laminin (green fluorescence) in a tissue section of 2 month denervated tibialis anterior muscle. Note the elements of cytoplasmic cross-striation visible in Nomarski contrast. Arrows show the location of two nuclei immunopositive for MyoD. Bar $10 \mu \mathrm{m}$ 
shown in Fig. 8b-d for cell culture). To visualize the boundaries and the topographical distribution of nascent muscle fibers in the tissue, we used double immunolabeling of longitudinal and transverse sections for the embryonic isoform of myosin and for laminin (Fig. 2ac). Embryonic muscle was used as a positive control for embryonic myosin expression, and virtually all embryonic muscle fibers reacted intensely with the antibody (Fig. 3c). Adult control muscle was practically immunonegative for this protein. Small foci of myogenesis, probably related to normal muscle growth and repair, were very rare (Fig. 2d).

In denervated muscle, dwarf longitudinally oriented myotubes immunopositive for embryonic myosin were closely associated with differentiated immunonegative muscle fibers (Fig. 2a-c). The presence of small myotubes significantly exceeded the control levels increasing 12- to 14-fold by the end of the first month following denervation (Table 1) and was a distinctive structural characteristic of long-term denervated muscle. Their number progressively increased during the first 2 months after denervation and constituted a 44-fold increase over the control. The presence of these structures decreased $30 \%$ and $60 \%$ from the level observed in 2 month denervated muscle after 4 and 7 months of denervation, respectively (Table 1 ). Most of the myotubes $(70-80 \%)$ immunopositive for embryonic myosin found in the denervated tissue were considerably smaller than normal adult, atrophic denervated and embryonic muscle fibers and did not express cross-striation (compare Fig. 2c and 3d to Fig. 3a-c). However, some of them attained or exceeded the linear dimensions typical of embryonic muscle fibers and had the elements of the cytoplasmic cross-striated pattern visible with Nomarski interference optics (Fig. 1b). Early formation of the basal lamina around small myotubes at the stage when they expressed the myogenic regulatory proteins and embryonic myosin (Figs. 1, 2b, c) appears to prevent additional rounds of myoblast fusions. Studies of adjacent serial sections have shown that most of the cytoplasm of dwarf muscle fibers was localized within 10 $12 \mu \mathrm{m}$ thick tissue sections and that they did not branch. Transverse sections of denervated muscle demonstrated that the cross-sectional areas of these myotubes on average comprised $20-60 \%$ of the cross-sectional areas of the fibers that are typical of fetal muscle.

To determine the mechanism of formation of dwarf muscle cells, we studied their fine structure using high resolution confocal and electron microscopy. Double labeling of the sections for the embryonic isotype of myosin and nuclear DNA has shown that the small myotubes, unlike differentiated multinucleated muscle fibers (Fig. 4a), contained typically no more than 2-4 nuclei. Confocal microscopy demonstrated that many of these cells were indeed binucleated (Fig. 4b), and our electron microscopic study clearly confirmed light microscopic observations (Fig. 5a, b). The ultrastructural analysis showed that small nascent myotubes have been frequently localized beneath the basal lamina of
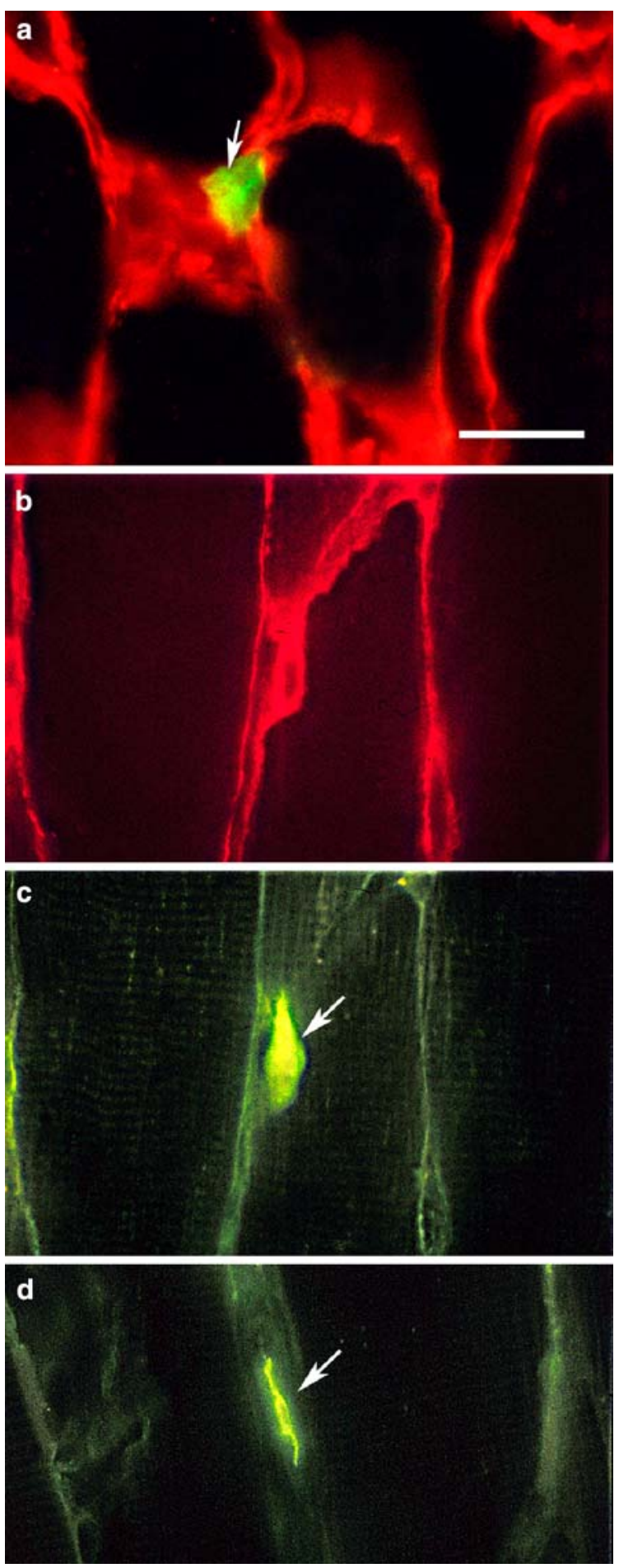

Fig. 2 a-d Localization of dwarf myotubes expressing embryonic myosin in denervated muscle. a Double immunolabeling of embryonic myosin (green fluorescence marked with the arrow) and laminin (red fluorescence) in the transverse section of 2 month denervated tibialis anterior muscle. b and $\mathbf{c}$ A longitudinal section of 1 month denervated muscle immunolabeled for laminin and embryonic myosin, respectively. The arrow in c indicates a diminutive myotube immunopositive for embryonic myosin in the area shown in $\mathbf{b}$. Note that the dwarf muscle fiber shown in $\mathbf{c}$ is completely separated from the surrounding muscle cells by the basal lamina. d A single differentiating muscle cell immunopositive for embryonic myosin in a control muscle (arrow). Bar $20 \mu \mathrm{m}$ 
Fig. 3 a-c Comparison of sizes of a normal terminally differentiated muscle fiber (a), a muscle fiber in 4 month denervated atrophic muscle (b), muscle fibers of 19 day-old rat embryo (c) and a dwarf muscle fiber in 2 month denervated tibialis anterior muscle (d) observed in longitudinal sections. Indirect immunofluorescent labeling for slow isotype of myosin $(\mathbf{a}, \mathbf{b})$ and the embryonic isoform of myosin (c, d). Arrows in c indicate individual muscle fibers, and the arrow in $\mathbf{d}$ shows the location of the dwarf muscle fiber located closely to muscle fibers immunonegative for embryonic myosin. Bar $30 \mu \mathrm{m}$
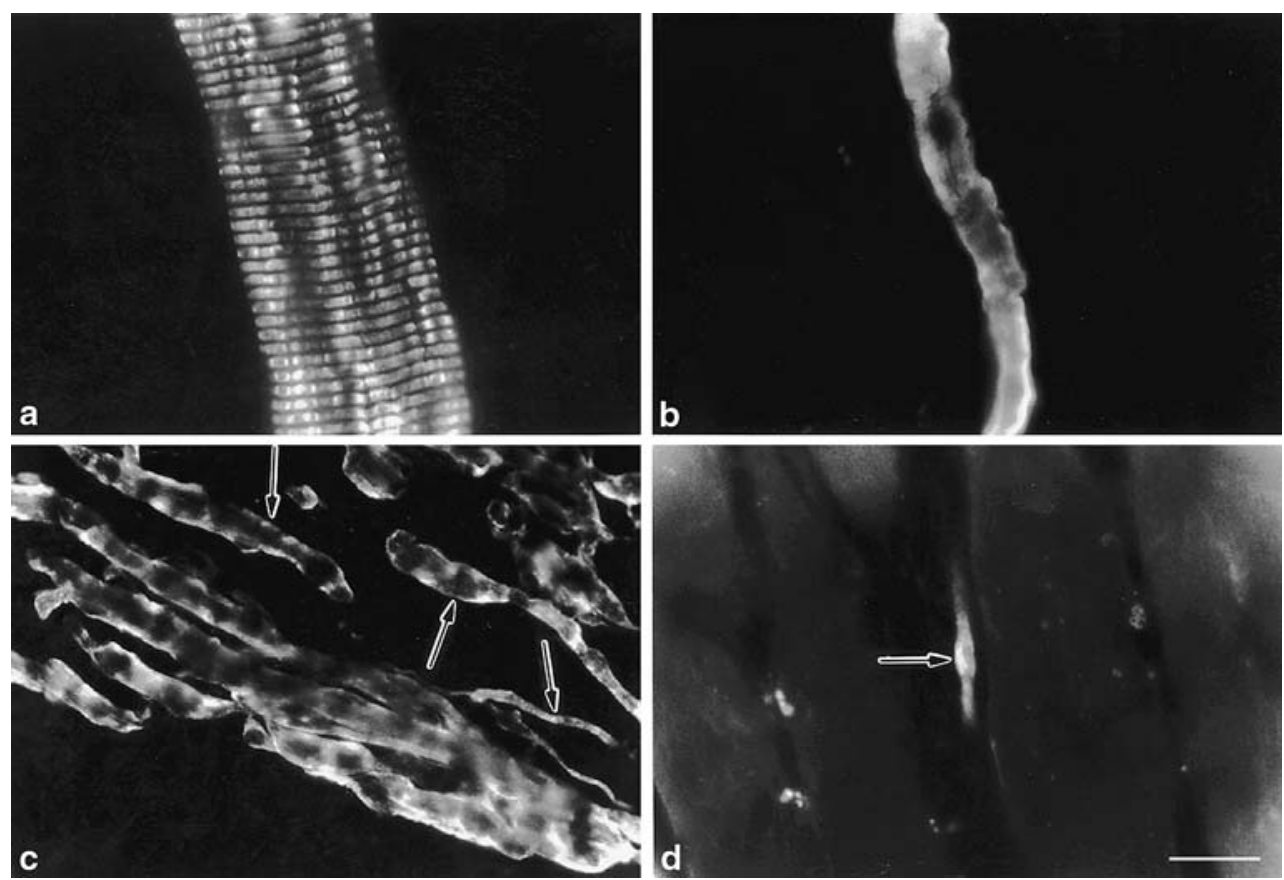

Table 1 Number of small myotubes expressing the embryonic isoform of myosin in control and long-term denervated tibialis anterior muscle

\begin{tabular}{lllllll}
\hline Time after denervation, months & Control & 0.5 & 1 & 2 & 4 \\
\hline Number of small myotubes, $\%$ & $0.07 \pm 0.03$ & $0.38 \pm 0.08^{*}$ & $0.94 \pm 0.11^{*}$ & $3.11 \pm 0.24 *$ & $2.17 \pm 0.17 *$ & $1.21 \pm 0.19 *$
\end{tabular}

Values are the mean \pm SD of the probes from four animals per each time point

*Statistically significant differences from the value observed in the control group, $P<0.05$

well differentiated muscle fibers (Fig. 5). Thus, they still occupied the location typical of satellite cells, which indicates that they appear to form by the fusion of two neighboring myoblasts or by the progeny of a satellite cell following single cell divisions. The nuclei in such cells were located either at a distance from each other (Fig. 5a), or in pairs close together (Fig. 5b). We did not find any intercellular contacts between the binucleated myogenic cells and their parent muscle fibers at the ultrastructural level. In some binucleated cells the central areas of the sarcoplasm contained cytoplasmic bridges still connecting the pairs of myoblasts following their fusion (Fig. 5a). Progressive accumulation of collagen lattice between muscle fibers accompanied the process of their post-denervation atrophy (Fig. 5).

To further investigate whether single fusions are involved in formation of binucleated cells and to visualize this process, we used immunolabeling of myogenic cells in tissue sections of denervated muscle for neural cell adhesion molecule (NCAM). NCAM is considered to be a marker of activated myogenic cells (Webster et al. 1988; Walsh et al. 1989; Irintchev et al. 1994) and is intensely expressed in the developing muscle (Covault and Sanes 1986). Immunolabeling for NCAM allowed us to demonstrate that the binucleated myotubes in denervated muscle did form as a result of single fusions of myoblasts (Fig. 6a-f). Figure 6 illustrates the consecutive stages of this process. Confocal microscopy of tissue sections immunolabeled for NCAM and laminin has shown that many myoblastic cells fused within the sublaminal space of atrophic muscle fibers (Fig. 7a-c). It is also important to mention that in 2 month denervated muscle, at the stage when myoblasts were abundant and the interstitial fibrosis did not reach an advanced level, we found the presence of nascent myotubes of the intermediate and standard sizes that were immunopositive for the embryonic proteins (not shown). This suggests that myoblasts in denervated muscle can initiate the process of normal regenerative myogenesis provided that they can find partners for fusion and do not have mechanical obstacles that restrict the regenerative response. Thus, in long-term denervated muscle, low local densities of activated satellite cells and the absence of a sufficient number of myoblasts available for fusion resulted in the formation of binucleated and trinucleated myotubes. This finding was further supported by the fact that in serial consecutive sections adjacent to the sections that contained closely apposed or fusing myoblasts (similar to ones shown in Fig. 7a-c), we did not find any additional NCAM-positive myogenic cells in many cases. In some areas no active myoblasts were present at the distance of up to $40-50 \mu \mathrm{m}$ above and below the 

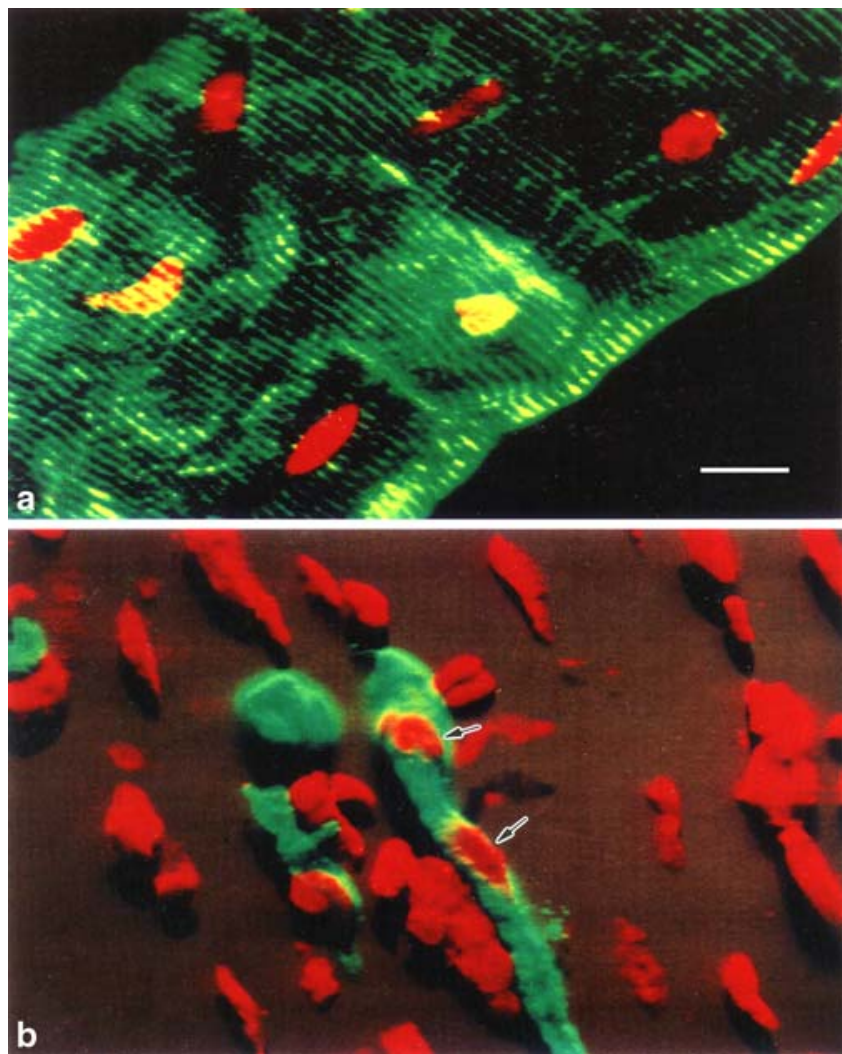

Fig. 4 a, b High resolution confocal digital images of a normal muscle fiber isolated from the extensor digitorum longus muscle (a) and a dwarf binucleated myotube in 2 month denervated extensor digitorum longus muscle (b). Indirect immunoflorescent staining (green fluorescence) for desmin (a) and embryonic isotype of myosin (b); nuclear DNA staining with propidium iodide (red fluorescence). Arrows in b indicate the location of two nuclei. Bar $10 \mu \mathrm{m}$

plain of sectioning. This situation was more typical of 4and 7 month denervated muscle that has already recruited large numbers of satellite cells in reparative myogenesis and therefore had a lower myoblast content. The structural characteristics of binucleated muscle cells at later stages of post-denervation atrophy were identical to those illustrated above for 2- and 4 month denervated tissue.

We also explored the possibility that long-term denervation considerably diminishes the intrinsic potential of myoblasts for fusion. To answer the question whether denervation impairs the capacity of myoblasts to fuse at the cellular level, we studied the formation of myotubes in cultures isolated from denervated and normal muscle. The goal of these experiments was to determine whether the formation of diminutive myotubes can be explained mainly by spatial separation of myogenic cells and by the lack of partners for fusion. A two-dimensional culture system allowed us to observe the boundaries of individual myoblasts and the number of nuclei in each differentiating cell following DNA staining with propidium iodide (Fig. 8b-d, 9a). Myo-
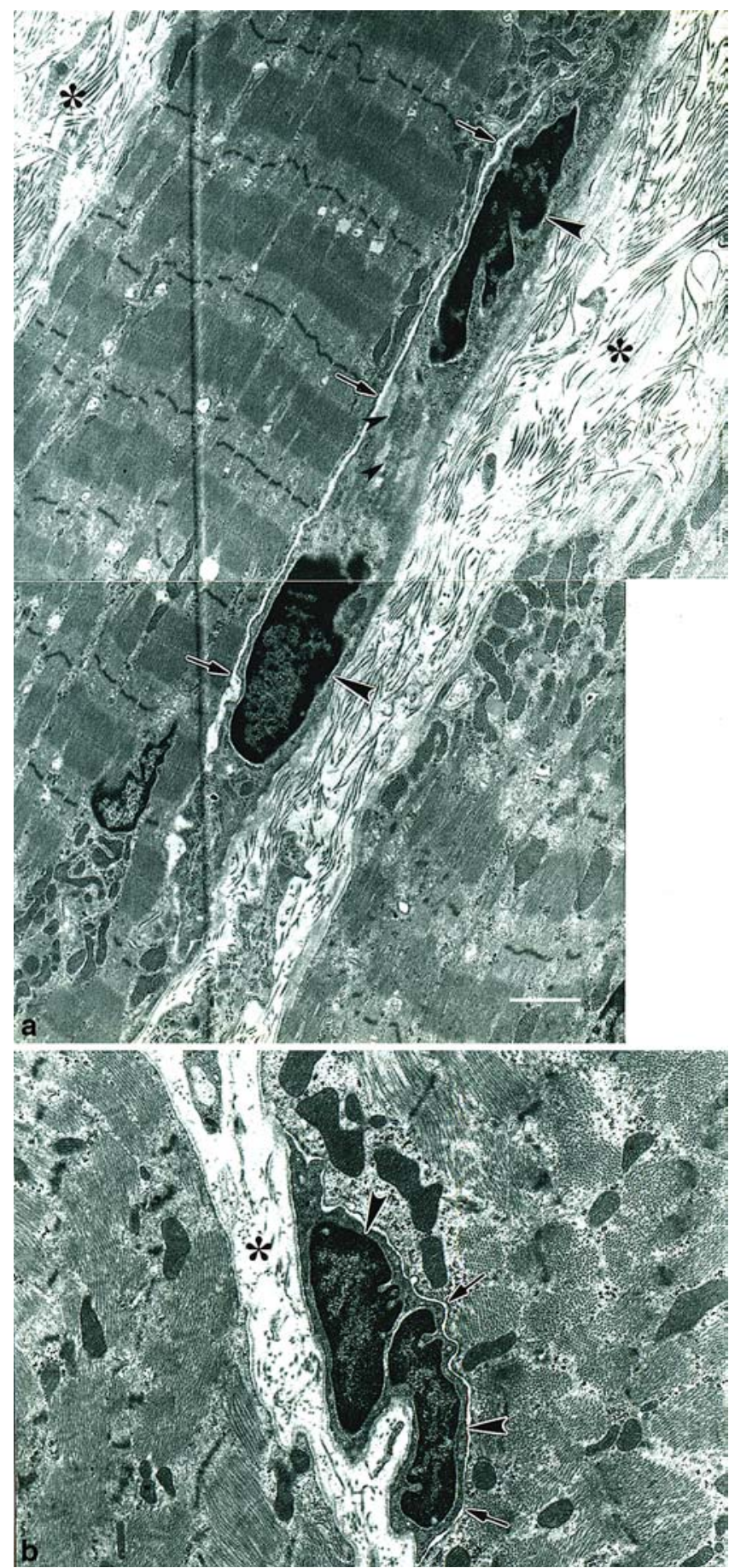

Fig. 5 a, b Electron microscopy of myotubes located beneath the basal lamina of the intact parent muscle fibers in denervated extensor digitorum muscle. Arrowheads indicate the typical locations of nuclei in binucleated cells: at some distance (a) or close to each other (b). Arrows show the sarcolemma of the parent fibers. Asterisks show the accumulation of collagen associated with the development of interstitial fibrosis. Small arrowheads in a indicate the cytoplasmic bridges in the area of cell fusion. Note the presence of well organized myofibrils in the parent cells, the absence of any intercellular contacts between muscle fibers and myotubes and developing interstitial fibrosis in the endomysium. a and $\mathbf{b}-4$ months and 2 months following denervation respectively. Bar $1 \mu \mathrm{m}$ 
Fig. 6 a-f Formation of dwarf myotubes in denervated muscle as a result of single fusions of activated satellite cells. a, c, Immunofluorescent labeling of myoblasts for NCAM. b, d Immunofluorescent labeling for laminin in the fields a and $\mathbf{c}$, respectively. The pattern of laminin localization shows the boundaries and orientation of muscle fibers. Double arrows in a show the closely apposed pairs of satellite cells. The cells in the pair on the left contact each other. A single arrow shows a binucleated cells immunopositive for NCAM, and the arrowhead shows a mononucleated myoblast located at a distance from other myoblastic cells. c Activated myoblasts extending elongated cytoplasmic processes processes to one another. e A binucleated cell immunopositive for

NCAM. f Staining for nuclear DNA with bisbenzimide in the field shown in e. Double arrows in $\mathbf{e}$ and $\mathbf{f}$ show the location of two nuclei in the NCAMpositive cell. a-f-4 month denervated tibialis anterior muscle. Bars $80 \mu \mathrm{m}(\mathbf{a}, \mathbf{b})$, $30 \mu \mathrm{m}(\mathbf{c}-\mathbf{f})$
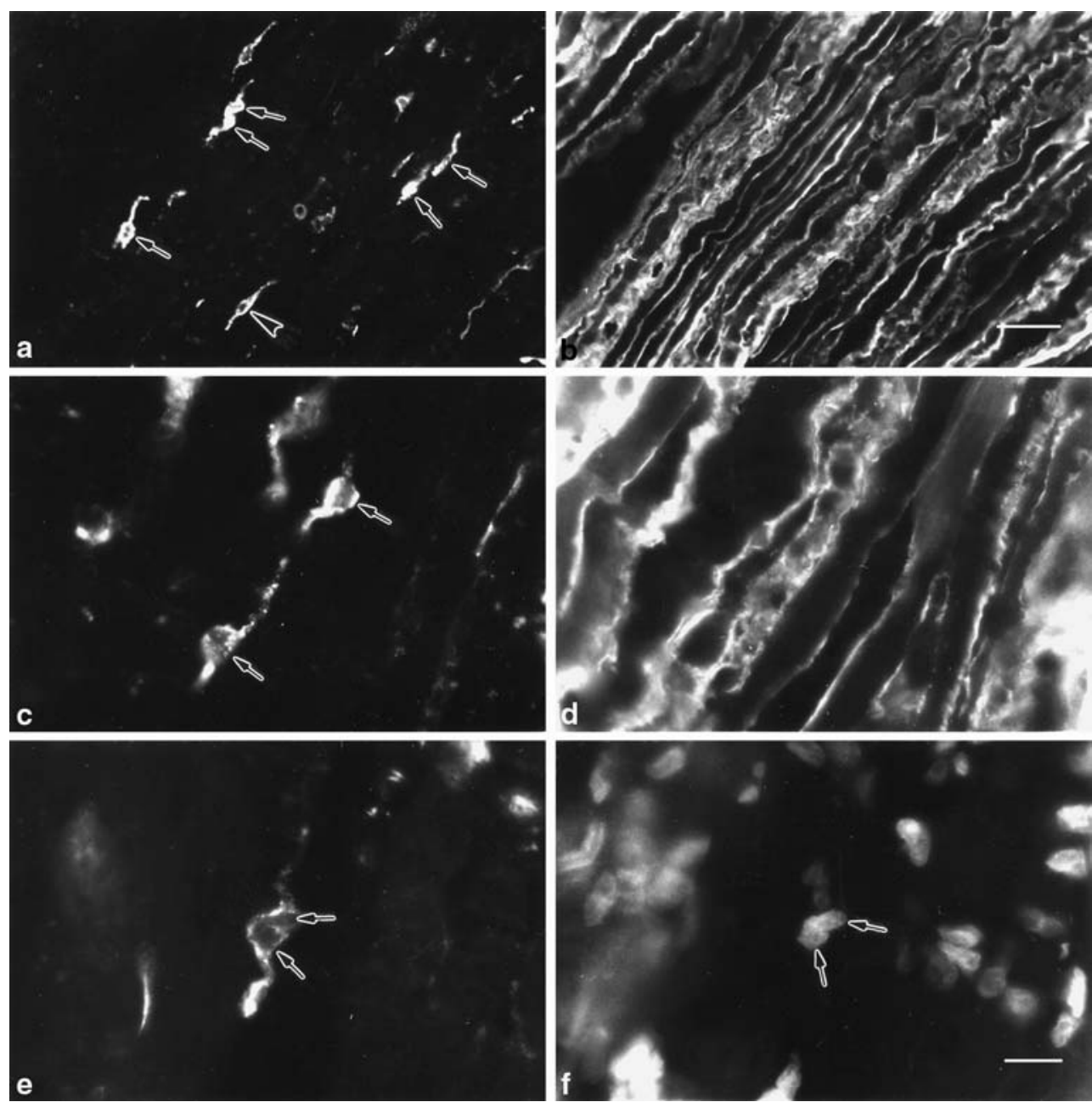

genic identity of the cells and homogeneity of the cultures was verified by immunofluorescent labeling for desmin, a tissue-specific intermediate filament protein expressed in the cells of the myogenic lineage (for literature see Sanger et al. 2004). The populations of myoblasts isolated from the control and denervated muscle consisted of mononucleated, desmin-positive cells (Fig. 8a). In both types of cultures, myoblasts successfully fused in the areas of increased cell densities, formed multinucleated myotubes and activated the expression of sarcomeric myosin (Fig. 9a, b). The fact that myogenic cells isolated from the denervated muscle were able to form multinucleated myotubes suggests that their capability for myogenic differentiation and fusion was not critically impaired after long-term denervation.

We found that in cultures plated at a low density (as described in the materials and methods), myoblasts from normal and denervated muscle expressed the markers of advanced myogenic commitment such as myogenin (Fig. 8b-d) and sarcomeric myosin (Fig. 9c) following single fusions. The structural characteristics of the dwarf myotubes, including the positions of their nuclei, in cell cultures were very similar to those observed in dener- vated muscle in situ (compare Figs. 8, 9 to Figs. 1, 2, 4b). As shown in Fig. 9c, a decrease of plating density in the cultures resulted in a considerable increase in the numbers of small mononucleated, binucleated and trinucleated cells immunopositive for sarcomeric myosin. The numbers of 1-4-nucleated myotubes were 6-8 times higher in both types of cultures plated at lower densities than in the cultures plated at a higher density that formed predominantly multinucleated myotubes. Thus, the myoblasts isolated from normal and denervated muscle and plated at low densities are able to activate synthesis of contractile proteins to a similar extent after single fusions or without fusion. The progressively increasing presence of actively proliferating fibroblasts among myogenic cells after 5-6 days in culture created a mechanical obstacle for successful fusions and appeared to be another contributing factor that prevented normal myogenesis. Taken together, these results suggest that in denervated muscle the mechanisms of myogenic commitment and myoblast fusion are not irreversibly blocked at the cellular level and that impaired regenerative myogenesis in situ is explained largely by the environment in the denervated tissue. 

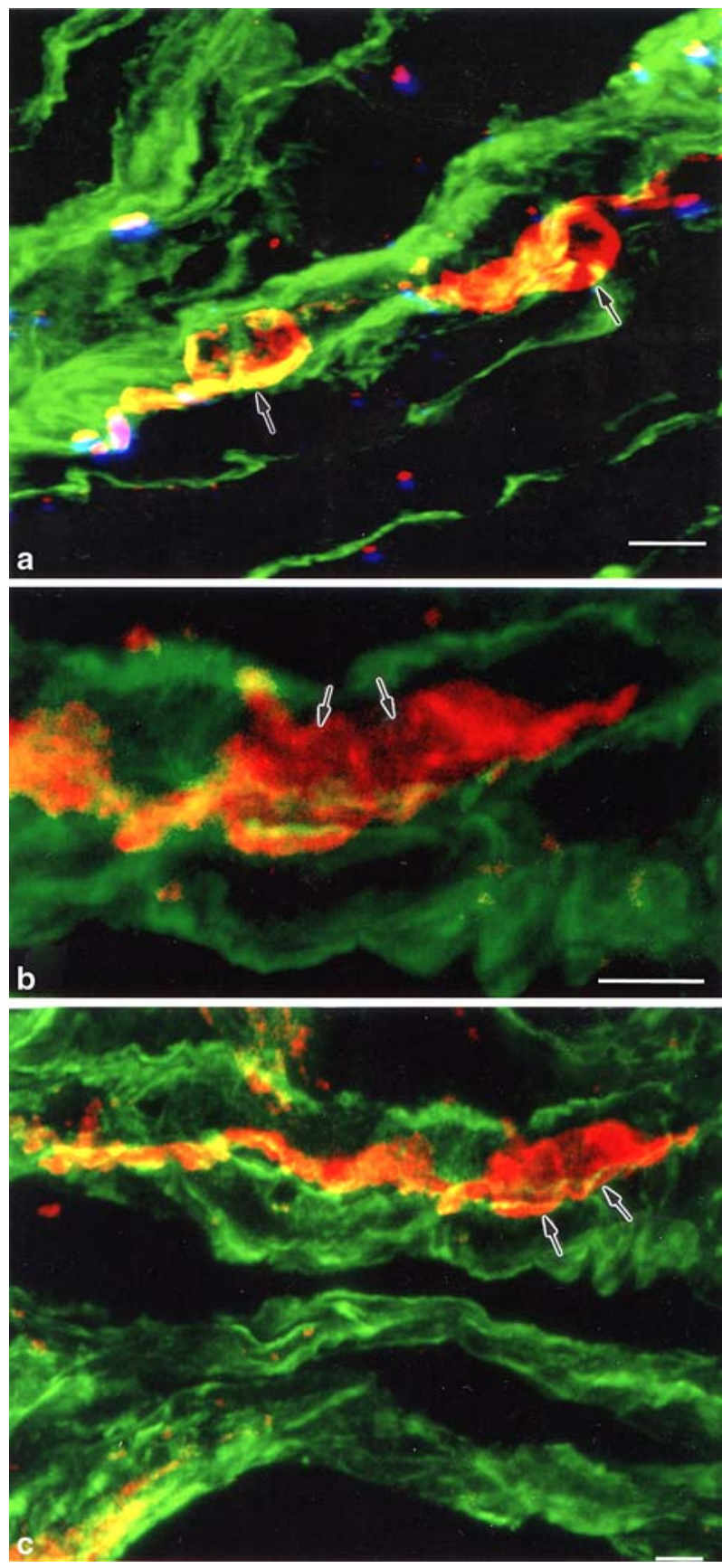

Fig. 7 a-c Confocal digital images of activated satellite cells fusing within the endomysial tubes of denervated muscle fibers. Double immunofluorescent labeling of myogenic cells for NCAM (red fluorescence) and for laminin (green fluorescence). a Two myogenic cells (arrows) located close to one another. b Two myogenic cells in the process of fusion (arrows). c The area shown in $\mathbf{b}$ (arrows) is presented at a lower magnification; note the absence of myogenic cells in the surrounding area. Bar $4 \mu \mathrm{m}$

\section{Discussion}

The regulatory mechanisms that control the response of satellite cells to denervation are still poorly studied. The available information concerns mostly the observations of the proliferative reaction of myogenic cells in denervated muscle and the changes in their number at different stages following denervation. ${ }^{3} \mathrm{H}$-thymidine autoradiography of muscle during the first week of denervation revealed the reactivation of DNA synthesis in the tissue, and it appears that at least some of DNA synthesizing nuclei belonged to satellite cells (for discussion see Murray and Robbins 1982; Snow 1983; McGeachie 1989). These data suggest that denervation may stimulate the return of myogenic precursor cells in the mitotic cycle. Counting of satellite cells in denervated muscle using electron microscopy demonstrated a 2- to 3-fold increase in their number during the first weeks following denervation (Vigue et al. 1997), which provides the direct evidence of satellite cell proliferation. Our earlier studies have shown that in the rat extensor digitorum longus muscle, the number of satellite cells related to the total number of nuclei had progressively decreased from $9.1 \pm 0.1 \%$ to $0.51 \pm 0.03 \%$ in 2 month denervated and 25 month denervated muscle, respectively (Vigue et al. 1997; Dedkov et al. 2001). A plausible explanation of this fact is that the precursor cells are involved in myogenesis, and their pool is gradually exhausting during the course of post-denervation muscle atrophy. At the same time the response of satellite cells appears to depend on the stage of development, species, muscle type and the experimental conditions (Bischoff and Francini-Armstrong 2004; Maier and Bornemann 2004). For example, denervation of neonatal rat limb muscles results in a sharp decrease in the number of satellite cells (Rodrigues Ade et al. 2002). As reported by Schmalbruch and Lewis (2000), in actively growing tissue of 6 week-old rats, the number of cell nuclei involved in DNA replication was significantly lower in the denervated soleus muscle than in the normal control, and the labeling indices did not exceed the control level in the extensor digitorum longus muscle.

Unlike the processes of embryonic development and regeneration, myogenesis in long-term denervated muscle continues for a long time and has a protracted and asynchronous character. The myogenic response is activated early following denervation and its initiation does not require severe atrophic changes or death of muscle fibers (for discussion see Borisov et al. 2001). There are indications that satellite cells in denervated muscle are involved in several structural types of regenerative reactions and that degeneration of muscle cells and capillaries in this experimental model occurs only at advanced stages of post-denervation atrophy (Borisov et al. 2000, 2001; Borisov and Carlson 2000). Interestingly enough, we found direct ultrastructural evidence of fusions of satellite cells with mature muscle fibers in long-term denervated muscle of adult rats (Borisov et al. 2005). This does not exclude the possibility that at least some myoblasts are able to fuse without re-entering the mitotic cycle. In this regard, it is important to mention that in regenerating adult chicken muscle, myogenic cells can fuse into myotubes after a single cell division in situ (Grounds and McGeachie 
Fig. 8 a-d Expression of early myogenic markers in cell cultures of myoblasts. a Immunolabeling for desmin in a 3 day culture from denervated muscle plated at a fusionpermissive density and consisting of mononucleated myogenic cells. b A binucleated myotube immunopositive for myogenin (green fluorescence in the nuclei located at a distance from one another) in a 4 day culture of normal muscle plated at a low density. Red

fluorescence shows the nuclei of myogenin-negative cells stained with propidium iodide. c Myogenin expression in a binucleated myogenic cell in a 6 day culture isolated from 2 month-denervated muscle and plated at a low density. d Counterstaining for nuclear DNA with propidium iodide in the field shown in c. Arrowheads in $\mathbf{c}$ and $\mathbf{d}$ indicate the location of two nuclei immunopositive for myogenin. Bars $60 \mu \mathrm{m}$ (a), $5 \mu \mathrm{m}(\mathbf{b}-\mathbf{d})$
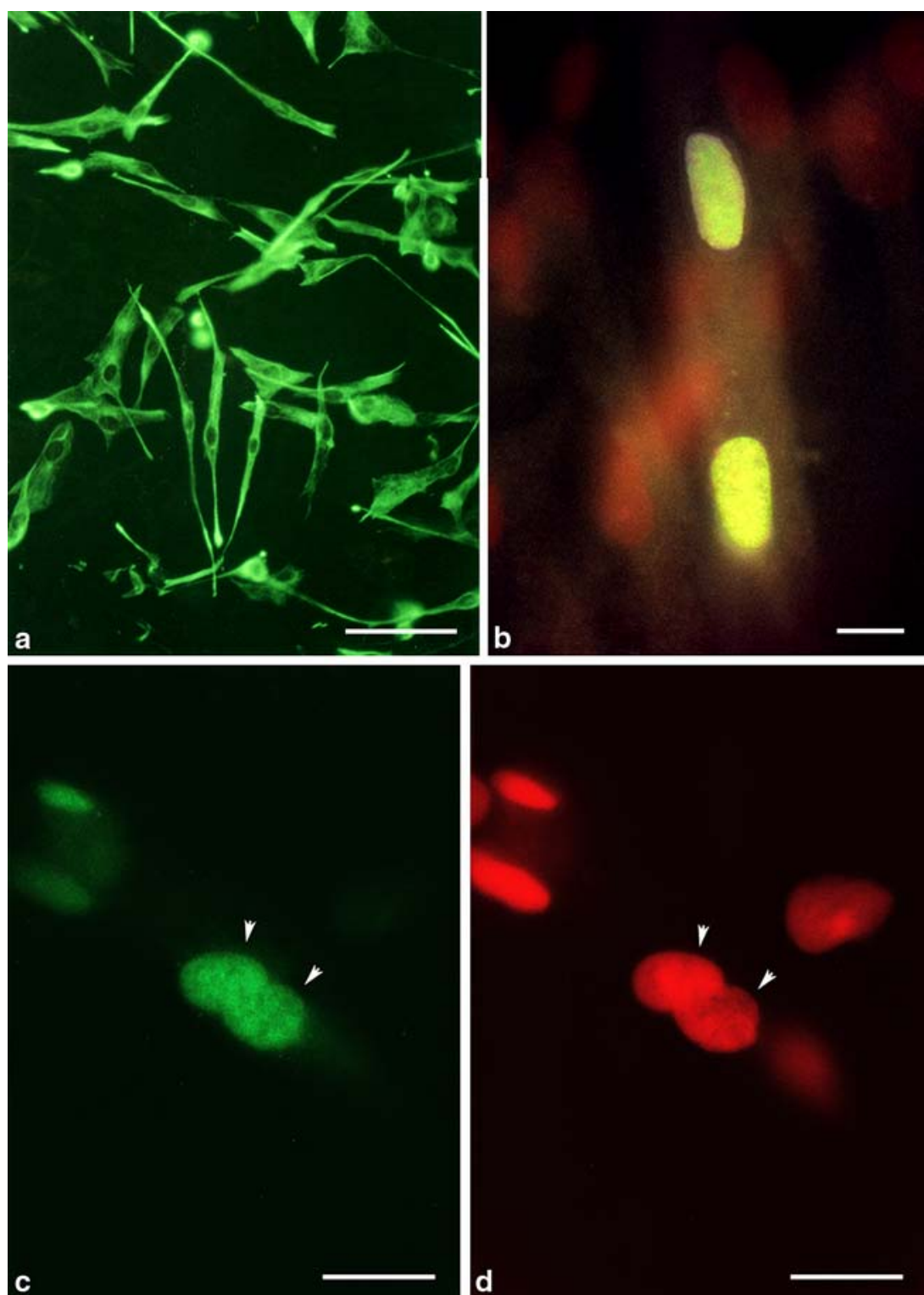

1989). The results of the present study show that satellite cells in denervated muscle are involved in a specific type of myogenesis that includes early expression of contractile proteins in diminutive myotubes following single myoblast fusions. The expression of the myogenic transcription factors is a critical event necessary for the commitment and differentiation of myogenic precusor cells during myogenesis in situ and in cell culture (Yablonka-Reuveni and Rivera 1994; Emerson and Hauschka 2004). The immunopositivity of some of diminutive myotubes for the myogenic regulatory factors suggests their recent formation or delayed progression toward terminal differentiation.

Skeletal muscle in mammals irreversibly loses the capacity to restore its normal function if reinnervation does not occur several months following denervation (Carlson and Faulkner 1988; Harrison 1989; Sunderland 1978; Carlson et al. 1996). It still remains unclear whether this process is determined by the intrinsic impairment of normal regenerative myogenesis at the cellular level, or whether the irreversibility of atrophy is explained by the involvement of the external factors, such as accumulation of collagen or inability of regenerating axons to restore normal functional relationship with atrophic muscle cells. Among these factors might be the absence of electric and trophic activity of the nerve, changes of the properties of the basal lamina or sarcolemma, modulations of local concentrations of growth promoting molecules, or the capacity to respond to mitogenic signals. It is also possible that the presence of intact muscle fibers and their direct contacts and regulatory interactions with satellite cells might restrict the proliferative response and affect differentiation of myoblasts. The basal laminae covering myogenic cells and small myotubes (Figs. 2, 5, 6, 7) separates them from other myoblasts and may act as a barrier that 

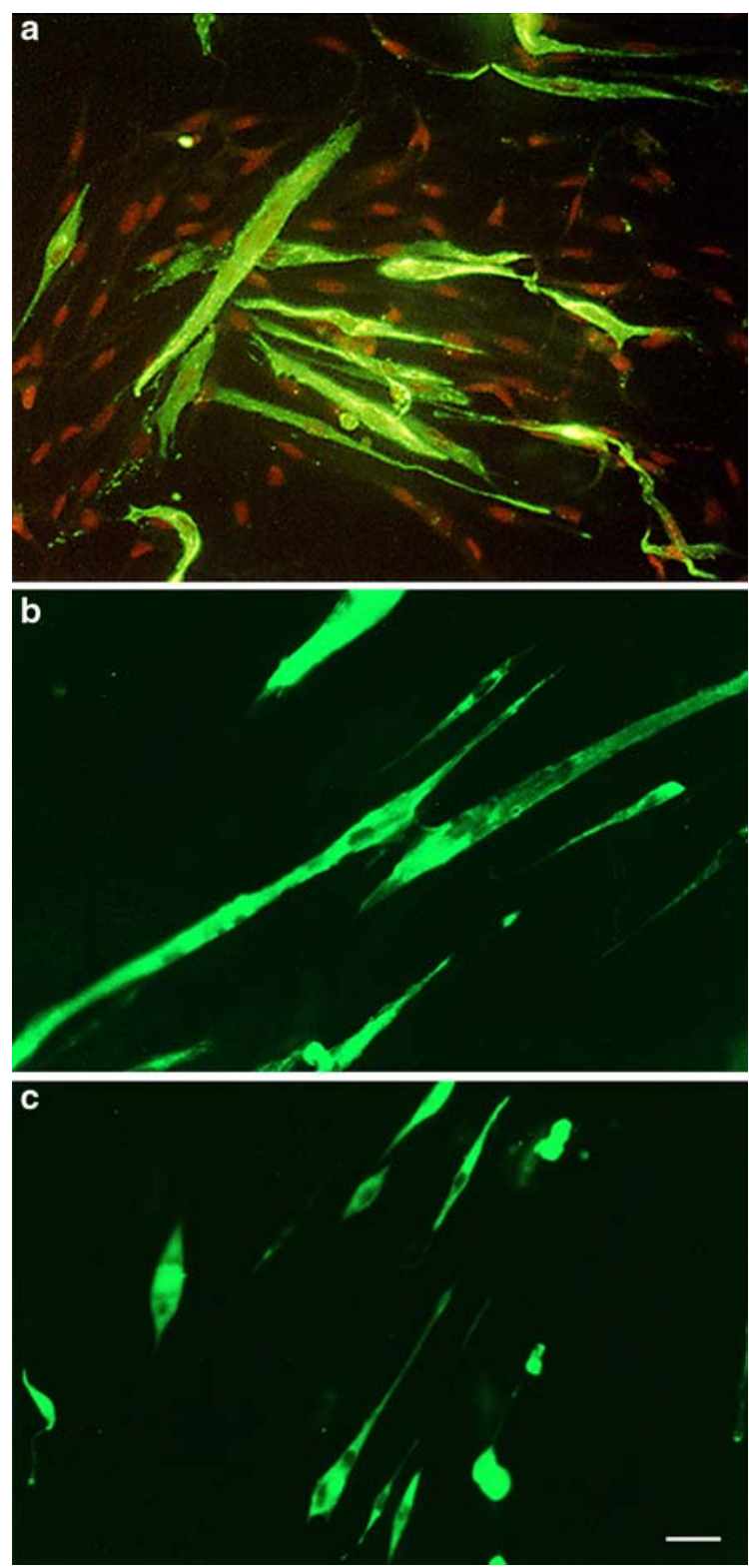

Fig. 9 a-c Density-dependent expression of sarcomeric myosin in mononucleated, binucleated and multinucleated myotubes in cultures of myogenic cells isolated from denervated (a) and innervated control tibialis anterior muscle $(\mathbf{b}, \mathbf{c})$. a Myotubes in a culture isolated from denervated muscle plated at the fusionpermissive density. Note the presence of mononucleated and binucleated myotubes in the areas of decreased local densities. Orange fluorescence visible between myotubes shows the localization of the nuclei of myosin-negative cells stained with propidium iodide. b Formation of myotubes in a cell culture of control muscle plated at the fusion-permissive density. c Formation of small mononucleated and binucleated myotubes immunopositive for sarcomeric myosin in the culture isolated from control muscle and plated at a low density. Bar $30 \mu \mathrm{m}$

prevents their fusions and access to mitogenic and humoral factors necessary for activation of the regenerative process. Our analysis of serial sections showed the absence of satellite cells above and below the pairs of fusing myoblasts and individual dwarf myotubes. This suggests that the progressive spatial separation of myo- genic cells does occur at least in some areas of long-term denervated tissue.

Unlike the situation in denervated muscle, during regeneration, when the normal structure of the tissue is damaged, satellite cells are able to undergo multiple repeated cell divisions and fusions leading to formation of multinucleated muscle fibers. It is important to mention that during the first several weeks after denervation, skeletal muscle is able to restore partially its functional capacity to a significant level following injury and regeneration, but the effect is temporary if reinnervation does not occur (Carlson et al. 1996). This suggests that when myogenic cells are released in the injured tissue, they are able to activate the regenerative process leading to a certain level of functional recovery.

The formation of multinucleated myotubes by the progeny of proliferating and fusing myogenic precursor cells have been traditionally considered as an obligatory prerequisite to normal muscle differentiation (reviewed by Sanger et al. 2004). The cellular mechanisms underlying the development of the competence for fusion still remain poorly studied. It was shown that chicken myogenic cells in vitro divide at least four times before they acquire the capacity to fuse into differentiating myotubes (Quinn et al. 1985). At the same time, another set of data indicates that fusion during muscle development, at least in some systems, appears to be a stochastic "opportunistic" process and that, depending on the local conditions, myoblasts can either proliferate or fuse (Konigsberg et al. 1978; Delvin and Konigsberg 1983; Konigsberg and Pfister 1986; Grounds and MCGeachie 1987). For this reason it is possible that the progressive inability to form large myotubes in denervated muscle is aggravated by the absence of the sufficient number of fusion-competent partners. Although denervation may affect the kinetics of myogenic commitment of satellite cells (Kuschel et al. 1999; Maier et al. 2002), our experiments with cultures of myoblasts isolated from normal and denervated muscle have shown that the capability of myoblasts for fusion was not irreversibly impaired following denervation. Further studies of the regulatory pathways that control the interrelations of the proliferative response and differentiation of satellite cells are important for understanding the mechanisms that underlie the impairment of the regenerative process during post-denervation muscle atrophy.

Acknowledgements Supported by NIH grant PO1-AG10821

\section{References}

Bischoff R (1990) Interaction between satellite cells and skeletal muscle fibers. Development 109:943-952

Bischoff R, Franzini-Armstrong C (2004) Satellite and stem cells in muscle regeneration. In: Engel AG, Franzini-Armstrong C (eds) Myology basic and clinical. McGraw-Hill, New York, pp 66-86

Borisov AB, Carlson BM (2000) Cell death in denervated skeletal muscle is distinct from classical apoptosis. Anat Rec 258:305318 
Borisov AB, Coro Antich RM, Rumyantsev PP (1985) DNA synthesis in cell cultures of atrial and ventricular cardiac myocytes. Tsitologiia 27:990-998

Borisov AB, Huang S-K, Carlson BM (2000) Remodeling of the vascular bed and progressive loss of capillaries in denervated skeletal muscle. Anat Rec 258:292-304

Borisov AB, Dedkov EI, Carlson BM (2001) Interrelations of myogenic response, progressive atrophy of muscle fibers, and cell death in denervated skeletal muscle. Anat Rtec 264:203-218

Borisov AB, Dedkov EI, Carlson BM (2005) Abortive myogenesis in denervated skeletal muscle: differentiation of satellite cells, their migration and block of terminal differentiation. Anat Embryol 209(4):269-279

Buckingham M (2001) Skeletal muscle formation in vertebrates. Curr Opin Genet Dev 11:440-448

Carlson BM (1991) Skeletal muscle regeneration. In: Oberpriller JO, Oberpriller JC, Mauro A (eds) The development and regenerative potential of cardiac muscle. Harwood Academic Publishers, New York, pp 439-454

Carlson BM, Faulkner JA (1988) Reinnervation of long-term denervated muscle freely grafted into an innervated limb. Exp Neurol 102:50-56

Carlson BM, Billington L, Faulkner JA (1996) Studies on the regenerative recovery of long-term denervated muscle in rats. Restor Neurol Neurosci 10:77-84

Charge SB, Rudnicki MA (2004) Cellular and molecular regulation of muscle regeneration. Physiol Rev 84:209-238

Chen EH, Olson EN (2004) Towards a molecular pathway for myoblast fusion in Drosophila. Trends Cell Biol 14:452-460

Covault J, Sanes J (1986) Distribution of N-CAM in synaptic and extrasynaptic portions of developing and adult skeletal muscle. J Cell Biol 102:716-730

Crameri RM, Langberg H, Magnusson P, Jensen CH, Schroder HD, Olesen JL, Suetta C, Teisner B, Kjaer M (2004) Chnages in satellite cells in human skeletal muscle after a single bout of high intensity exercise. J Physiol 558:333-340

Dedkov EI, Kostrominova TY, Borisov AB, Carlson BM (2001) Reparative myogenesis in long-term denervated skeletal muscles of adult rats results in a reduction of the satellite cell population. Anat Rec 263:139-154

Delvin BH, Konigsberg IR (1983) Reentry into the cell cycle of differentiated skeletal myocytes. Dev Biol 95:175-192

Emerson CP, Hauschka SD (2004) Embryonic origin of skeletal muscles. In: Engel AG, Granzizi-Armstrong C (eds) McGrowHill, New York, pp 3-44

Grounds MD, McGeachie JK (1987) A model of myogenesis in vivo, derived from detailed autoradiographic studies of regenerating skeletal muscle, challenges the concept of quantal mitosis. Cell Tissue Res 250:563-569

Grounds MD, McGeachie JK (1989) Myogenic cells of regenerating adult chicken muscle can fuse into myotubes after a single cell division in vivo. Exp Cell Res 180:429-439

Harrison D (1989) Current trends in the treatment of established unilateral cerebral palsy. In: Karcher E (ed) Functional surgery of the head and neck. Graz, Austria, pp 9-16

Hawke TJ, Garry DJ (2001) Myogenic satellite cells: physiology to molecular biology. J Appl Physiol 91:534-551

Horsley V, Pavlath GK (2004) Forming a multinucleated cell: molecules that regulate myoblast fusion. Cells Tissues Organs 176:67-78

Irintchev A, Zeschnigk M, Starzinski-Powitz A, Wernig A (1994) Expression pattern of M-cadherin in normal, denervated, and regenerating mouse muscles. Dev Dyn 199:326-337

Konigsberg IR, Pfister KK (1986) Replicative and differentiative behavior in daughter pairs of myogenic stem cells. Exp Cell Res 167:63-74

Konigsberg IR, Sollmann PA, Mixter LO (1978) The duration of the terminal G1 of fusing myoblasts. Dev Biol 63:11-26
Kuschel R, Yablonka-Reiveni Z, Bornemann A (1999) Satellite cells on isolated myofibers from normal and denervated adult rat muscle. J Histochem Cytochem 47:1375-1383

Maier A, Bornemann A (2004) M-cadherin transcription in satellite cells from normal and denervated muscle. Am J Physiol Cell Physiol 286:C708-C712

Maier A, Zhou Z, Bornemann A (2002) The expression profile of myogenic transcription factors in satelliote cells from denervated rat muscle. Brain Pathol 12:170-177

Matecki S, Guibinga GH, Petrof BJ (2004) Regenerative capacity of the dystrophic (mdx) diaphragm after induced injury. Am J Physiol 287:R961-R968

McGeachie JK (1989) Sustained cell proliferation in denervated skeletal muscle of mice. Cell Tissue Res 257:455-457

Mesires NT, Doumit ME (2002) Satellite cell proliferation and differentiation during postnatal growth of porcine skeletal muscle. Am J Physiol 282:C899-C906

Morgan JE, Partridge TA (2003) Muscle satellite cells. Intern J Biochem Cell Biol 35:1151-1156

Murray MA, Robbins N (1982) Cell proliferation in denervated muscle: identity and origin of dividing cells. Neuroscience 7:1823-1833

Paoni NF, Peale F, Wang F, Errett-Baroncini C, Steinmetz H, Toy K, Bai W, Williams PM, Bunting S, Gerritsen ME, PowellBraxton L (2002) Time course of skeletal muscle repair and gene expression following acute hind limb ischemia in mice. Physiol Genom 11:263-272

Pownall ME, Gustaffsson MK, Emerson CP (2002) Myogenic regulatory factors and the specification of muscle progenitors in vertebrate embryos. Ann Rev Cell Dev Biol 18:747-783

Quinn LS, Nameroff M, Holtzer H (1985) Generation of chick skeletal muscle cells in groups of 16 from stem cells. Nature 313:692-694

Reynolds ES (1963) The use of lead citrate at high $\mathrm{pH}$ as an electron-opaque stain in electron microscopy. J Cell Biol 17:208213

Rodrigues Ade C, Geuna S, Rodrigues SP, Silva MD, Aragon FF (2002) Satellite cells and myonuclei in neonatally denervated rat muscle. Ital J Anat Embryol 107:51-56

Sanger JW, Sanger JM, Franzini-Armstrong C (2004) Assembly of the skeletal muscle cell. In: Engel AG, Franzini-Armstrong C (eds) Myology basic and clinical. McGraw-Hill, New York, pp 45-65

Schmalbruch H, Lewis DM (2000) Dynamics of nuclei of muscle fibers and connective tissue cells in normal and denervated rat muscles. Muscle Nerve 23:617-626

Snow MH (1983) A quantitative ultrastructural analysis of satellite cells in denervated fast and slow skeletal muscles of the mouse. Anat Rec 207:593-604

Sunderland S (1978) Nerve and nerve injuries, 2nd edn. ChurchillLivingston, Edinburgh

Vigue CA, Lu D-X, Huang S-K, Rengen H, Carlson BM (1997) Quantitative study of the effects of long-term denervation on the extensor digitorum longus muscle of the rat. Anat Rec 248:346-354

Walsh FS, Dickinson G, Moore SE, Barton CH (1989) Unmasking N-CAM. Nature 339:516

Webster C, Pavlath GK, Parks DR, Walsh FS, Blau HM (1988) Isolation of human myoblasts with the fluorescence-activated cell sorter. Exp Cell Res 174:252-265

Yablonka-Reuveni Z, Paterson BM (2001) MyoD and myogenin expression patterns of fetal and adult chicken myoblasts. J Histochem Cytochem 49:455-462

Yablonka-Reuveni Z, Rivera AJ (1994) Temporal expression of regulatory and structural muscle proteins during myogenesis of satellite cells on isolated adult rat fibers. Devel Biol 164:588-603 